\title{
Hematological and liver toxicity of anti-tuberculosis drugs
}

\author{
Maryam-Sadat Mirlohi ${ }^{1}$, Alireza Ekrami ${ }^{2}$, Saeed Shirali $^{3}$, Mehdi Ghobeishavi ${ }^{4}$, Fatemeh Pourmotahari ${ }^{5}$
}

\footnotetext{
${ }^{1}$ M.Sc. of Biochemistry, Student Research Committee, Department of Clinical Biochemistry, Ahvaz Jundishapur University of Medical Sciences, Ahvaz, Iran

${ }^{2}$ Ph.D. of Microbiology, Faculty Member, Health Research Institute, Infectious and Tropical Diseases Research Center, Ahvaz Jundishapur University of Medical Sciences, Ahvaz, Iran

3 of Biochemistry, Faculty Member, Research Center of Thalassemia and Hemoglobinopathy, Department of Laboratory Sciences, School of Paramedical Sciences, Ahvaz Jundishapur University of Medical Sciences, Ahvaz, Iran

${ }^{4}$ Graduate Degree of Laboratory Sciences, Student Research Committee, Department of Clinical Biochemistry, Ahvaz Jundishapur University of Medical Sciences, Ahvaz, Iran

${ }^{5}$ M.Sc. of Biostatistics, Department of Biostatistics Sciences, Dezful University of Medical Sciences, Dezful, Iran
}

\section{Type of article: Original}

\begin{abstract}
Introduction: Tuberculosis (TB) is a major global health problem, and anti-tuberculosis drugs can cause severe adverse reactions. The aim of this study was to determine hematological and biochemical changes and associated risk factors in smear positive pulmonary tuberculosis patients undergoing treatment with standard protocols.

Methods: In a descriptive study, a total of 40 tuberculosis patients aged between 15-60 years were collected from hospitals in Khuzestan Province (Iran) from March 2013 to March 2014. The patients were treated with drugs (isoniazid, rifampicin, ethambutol, and pyrazinamide) during the initial two months, followed by isoniazid and rifampicin for the next four to six months. Activities of liver enzymes (ALT, AST, and ALP) and hematological parameters were recorded before and after treatment. Data were analyzed using paired samples t-test and Wilcoxon test by SPSS 16.

Results: After using drug treatments, hematological parameters (RBC, $\mathrm{Hb}, \mathrm{HCT}, \mathrm{MCV}, \mathrm{MCH}$, and MCHC), except platelet count, were changed significantly $(\mathrm{p} \leq 0.001)$. Liver enzyme activities (ALT, AST, and ALP) were decreased significantly $(\mathrm{p} \leq 0.001)$ after treatment.

Conclusion: In this study, changes of hematological and biochemical parameters have been observed in patients with pulmonary tuberculosis. It can be concluded that the anti-tuberculosis treatment is associated with changes of hematological parameters and liver enzymes.
\end{abstract}

Keywords: Tuberculosis, Hematological parameters, Liver enzymes, Isoniazid, Khuzestan

\section{Introduction}

Tuberculosis (TB) is one of the most serious infectious diseases worldwide and a leading cause of death for nearly 3 million deaths annually. In 2013, an estimated 9.0 million people were infected with tuberculosis, and 1.5 million died from this disease. In Iran, TB incidence was considerably lower than in neighboring countries over the last decade (1). TB is a highly prevalent chronic devastating disease caused by Mycobacterium tuberculosis. This bacteria is a sedentary, obligate aerobic, acid fast facultative intracellular rod-shaped bacterium, with long generation time and preference to localize in macrophage and typically affects the lungs (pulmonary TB) but can affect other sites as well as extra pulmonary TB $(2,3)$. The most common method to detect TB is sputum smear microscopy. In this method, bacteria are observed in sputum samples examined under a microscope. In areas with more equipped laboratories, cases of TB also are diagnosed via culture methods (the reference standard method).

\section{Corresponding author:}

Dr. Saeed Shirali, Research Center of Thalassemia and Hemoglobinopathy, Ahvaz Jundishapur University of Medical Sciences, Ahvaz, Iran. Tel: +98.9124350966, Email: saeed.shirali@gmail.com

Received: February 07, 2016, Accepted: August 24, 2016, Published: September 2016

iThenticate screening: July 19, 2016, English editing: September 04, 2016, Quality control: September 18, 2016

(C) 2016 The Authors. This is an open access article under the terms of the Creative Commons Attribution-NonCommercialNoDerivs License, which permits use and distribution in any medium, provided the original work is properly cited, the use is non-commercial and no modifications or adaptations are made. 
Following recent development in TB diagnostics, rapid molecular tests have been used for diagnosis of TB and drug-resistant TB (4). Treatments of tuberculosis involve the use of a mixture of drugs to hinder drug resistance. A composition of drugs is highly effective in treating tuberculosis, but it may also lead to enhanced toxicity and side effects. TB is treated with an initial intensive 2-month regimen consisting of multiple antibiotics, including rifampicin (RIF), isoniazid (INH), pyrazinamide (PZA), and ethambutol (EMB) or streptomycin (SM) to guarantee that mutants resistant to a single drug will not occur $(5,6)$. The four-drug combination therapy (isoniazid [INH], rifampin [RIF], pyrazinamide [PZA], and ethambutol [EMB]) is the main treatment of active tuberculosis, of which the first three are hepatotoxic. Multidrug-resistant strains, resistant to at least isoniazid and rifampicin, requires our attention because they requires the use of second-line drugs that are difficult to procure and are much more toxic and expensive than the first-line regimen $(7,8)$. Indicators of hepatocellular functions that usually are assayed in clinical toxicology studies are the enzymes alanine aminotransferase (ALT) and aspartate aminotransferase (AST). Changes in concentrations of the exudation enzymes ALT and AST show changes in cell membrane function, such as changes in permeability or the more obvious cell membrane disconnection $(5,9)$. In tuberculosis, many hematological and biochemical abnormalities are common, and they are valuably aiding to patients' management and diagnosis. Tuberculosis patients may have a rise in hemoglobin and hematocrit levels due to the response to treatment. Hematological abnormalities associated with TB infection have, to the best of our knowledge, been incompletely investigated $(4,5)$. As a nonspecific test for inflammatory and infectious processes, erythrocyte sedimentation rate (ESR) is used to evaluate activity level and disease extent, response to treatment, and the prognosis of subacute and chronic diseases such as tuberculosis. The reduction in this test was regarded as a good index to observe the drug response and disease progress or regress $(10,11)$. In line with our recent studies on the effect of different compounds on liver function and blood factors (12-16), this study was designed to observe the hematological and biochemical changes and associated risk factors in smear positive pulmonary tuberculosis patients undergoing treatment with standard protocol.

\section{Material and Methods}

\subsection{Study design and setting}

This descriptive study was conducted on patients with pulmonary tuberculosis who were treated with standard protocol in the hospitals of Khuzestan Province (Iran) from March 2013 to March 2014. A total of 40 male and female tuberculosis patients aged 15-60 years were included in this study. Subjects were selected from among smear positive pulmonary tuberculosis patients who were referred to TB reference of Khuzestan province. Exclusion criteria were HIV positive, pregnancy, chronic and acute liver disease, and multidrug-resistant tuberculosis patients. The drug regimens were isoniazid, rifampicin, ethambutol and pyrazinamide during the initial two months, followed by isoniazid and rifampicin for the next four to six months $(5,12)$. Patients with pulmonary tuberculosis were selected among patients with tuberculosis reference laboratory province. Exclusion criteria were the presence of diseases other than tuberculosis (like HIV). Also patients with drug-resistant tuberculosis were excluded from the study.

\subsection{Blood collection and processing}

After identification, about 3-4 ml peripheral venous blood was drawn aseptically by sterile syringe: $2 \mathrm{ml}$ was transferred into a tube containing $0.2 \mathrm{ml}$ of $4 \%$ ethylene diamine tetra acetic acid (EDTA) solution, and then it was analyzed in the hematology analyzer (Sysmex, KX-21) for evaluation of different blood parameters including hemoglobin (Hb); total leukocyte count (TLC); and differentiation neutrophils; monocytes and lymphocytes; red blood cell(RBC); platelet count; hematocrit (HCT); mean corpuscular volume (MCV); mean corpuscular hemoglobin $(\mathrm{MCH})$; mean corpuscular hemoglobin concentration $(\mathrm{MCHC})$; in addition, $2 \mathrm{ml}$ of the blood were used for erythrocytic sedimentation rate (ESR) determination.

\subsection{Liver function test}

The routine laboratory testing was performed at the Central Pathology Laboratory, including AST, ALT, and alkaline phosphatase (ALP) as a liver function test.

\subsection{Statistical analysis}

Data is presented as mean \pm Standard Deviation (S.D.) and were compared using the paired samples t-test for continuous variables and Wilcoxon test. SPSS version 16.0 was used for analyzing the data, and p-values $\leqslant 0.05$ were considered significant. 


\section{Results}

A total of 40 male and female smear positive pulmonary tuberculosis patients from the TB reference laboratory were selected to evaluate different hematological parameters and some liver function enzymes. About $70 \%$ of patients over 50 years old, $30 \%$ were children, and about $80 \%$ were men. As shown in Table 1, there are significant changes in enzymatic and hematological parameters of tuberculosis patients before and after the treatment course. The mean of hematological parameter ( $\mathrm{RBC}, \mathrm{Hb}, \mathrm{HCT}, \mathrm{MCV}, \mathrm{MCH}$, and $\mathrm{MCHC}$ ) in the study groups was significantly increased in patients after using the drugs, in comparison to before using the drugs $(\mathrm{p}<0.001)$ (Table 1).

Table 1. Changes in serum parameters in TB patients before and after treatments.

\begin{tabular}{|l|l|l|l|l|}
\hline Parameters & Pre $($ Mean $\pm \mathrm{SD})$ & Post $($ Mean $\pm \mathrm{SD})$ & Pre-Post $($ Mean $\pm \mathrm{SD})$ & $p$-value \\
\hline $\mathrm{WBC}\left(\times 10^{9} \mathrm{~L}\right)$ & $9.85 \pm 2.41$ & $8.05 \pm 1.56$ & $1.79 \pm 1.42$ & 0.000 \\
\hline $\mathrm{RBC}\left(\times 10^{12} \mathrm{~L}\right)$ & $4.87 \pm 0.46$ & $4.92 \pm 0.48$ & $0.49 \pm 0.72$ & 0.000 \\
\hline $\mathrm{Hb}(\mathrm{g} / \mathrm{dl})$ & $12.92 \pm 1.44$ & $13.45 \pm 1.31$ & $0.53 \pm 0.49$ & 0.000 \\
\hline $\mathrm{Hct}(\%)$ & $39.00 \pm 3.66$ & $40.35 \pm 3.77$ & $1.35 \pm 0.88$ & 0.000 \\
\hline $\mathrm{MCV}(\mathrm{fl})$ & $80.71 \pm 9.26$ & $81.87 \pm 8.95$ & $1.16 \pm 1.11$ & 0.000 \\
\hline $\mathrm{MCH}(\mathrm{pg})$ & $26.75 \pm 3.40$ & $27.62 \pm 3.22$ & $0.87 \pm 0.70$ & 0.000 \\
\hline $\mathrm{MCHC}(\mathrm{g} / \mathrm{dl})$ & $33.12 \pm 1.29$ & $33.84 \pm 1.34$ & $0.72 \pm 0.55$ & 0.000 \\
\hline Plt $\left(\times 10^{9} \mathrm{~L}\right)$ & $294.17 \pm 91.97$ & $303.42 \pm 98.42$ & $9.25 \pm 34.10$ & 0.094 \\
\hline Neu $(\%)$ & $65.97 \pm 9.77$ & $60.05 \pm 6.59$ & $5.92 \pm 6.99$ & 0.000 \\
\hline Lym $(\%)$ & $33.92 \pm 9.68$ & $21.05 \pm 6.04$ & $14.67 \pm 8.23$ & 0.002 \\
\hline ESR $(\mathrm{mm} / \mathrm{hr})$ & $35.72 \pm 10.83$ & $37.80 \pm 8.45$ & $3.87 \pm 7.51$ & 0.000 \\
\hline AST $(\mathrm{IU} / \mathrm{L})$ & $44.47 \pm 13.37$ & $28.22 \pm 8.14$ & $16.25 \pm 9.64$ & 0.000 \\
\hline ALT $(\mathrm{IU} / \mathrm{L})$ & $40.22 \pm 13.40$ & $27.05 \pm 7.26$ & $13.17 \pm 10.59$ & 0.000 \\
\hline ALP $(\mathrm{IU} / \mathrm{L})$ & $208.82 \pm 34.01$ & $160.15 \pm 21.20$ & $48.67 \pm 23.90$ & 0.000 \\
\hline
\end{tabular}

WBC: white blood cell; RBC: red blood cell; Hb: hemoglobin; HCT: hematocrit; MCV: mean corpuscular volume; MCH: mean corpuscular hemoglobin; MCHC: mean corpuscular hemoglobin concentration; Plt: platelet count; Neu: neutrophils; Lym: lymphocytes; ESR: erythrocytic sedimentation rate; AST: aspartate aminotransferase; ALT: alanine aminotransferase; ALP: alkaline phosphatase

The mean of the $\mathrm{Hb}$ was significantly increased in patients after using drugs $(13.45 \pm 1.31)$ compared with before using drugs $(12.92 \pm 1.44)$. Also the hematocrit percentage was increased in the patient to after using drugs (40.35 \pm 3.77) compared with before using the drugs $(39.00 \pm 3.66)$. The WBC and platelet counts, leukocyte count, and neutrophil count of tuberculosis patients were investigated before and after using drug. The mean of WBC before using drugs was $9.85 \pm 2.41$ and after drugs was $8.05 \pm 1.56$. Before using the drugs, neutrophil was $65.97 \pm 9.77$; after using drugs, it was $60.05 \pm 6.59$, which shows a significant decrease $(\mathrm{p}<0.001)$. Before using the drugs, Lym was $33.92 \pm 9.68$; after using drugs, it was $37.80 \pm 8.45$, which shows a significantly increase $(\mathrm{p}<0.01)$ (Table 1$)$. According to Table 1, in this study, platelet count before using drugs was $294.17 \pm 91.97$; after using drugs, it was $303.42 \pm 98.42$; thus, difference was not significant. In this work, it has been found that the values of ESR for tuberculosis patients before using drugs $(35.72 \pm 10.83)$ are highly significantly increased $(\mathrm{p}<0.001)$ compared with after using drugs $(21.05 \pm 6.04)$ (Table 1$)$. The present study showed that there was a significant decrease $(\mathrm{p}<0.001)$ in liver enzymes. ALP before using the drug was $208.82 \pm 34.01$; after using the drug, it was $160.15 \pm 21.20$. AST before using the drug was $44.47 \pm 13.37$; after using the drug, it was $28.22 \pm 8.14$. ALT before the drug was $40.22 \pm$ 13.40; after using the drug, it was $27.05 \pm 7.26$.

\section{Discussion}

Tuberculosis remains a main public health problem in developing countries, as it is the largest cause of death in the world as an individual infectious disease. There is not much literature about the hematological parameters in pulmonary TB patients in Iran's population $(17,18)$. This study reveals the hematological profile and some biochemical parameters in pulmonary tuberculosis patients. As shown in the results section, the values of red cell parameters - including $\mathrm{RBC}, \mathrm{Hb}, \mathrm{HCT} \mathrm{MCV}, \mathrm{MCH}$, and $\mathrm{MCHC}$ - were significantly increased in patients after treatment. This finding is similar to studies by Araujo et al., Subodh kumar et al., and Shafee et al., which showed decreased levels of hemoglobin in patients $(19,20)$. Some previous studies conducted by Lombard and Mansvelt and Charles et al., also reported anemia in most tuberculosis patients $(16,19)$. It has been reported that lower MCHC values may be associated with deficiency of iron, which may be acquired through extrinsic factors $(4,20)$. Kumar Thatoi et al. have reported the tumor necrosis factor- $\alpha$ (TNF- $\alpha$ ), and other cytokines released by activated 
monocytes suppress the erythropoietin production leading to anemia (20-22). This decrease in hemoglobin also might be due to the effects of anti-tuberculosis drugs during the period of treatment (2). Tengku et al. found that the majority of patients had normocytic normochromic anemia with thrombocytosis. These findings are similar to a study by Morris et al. who found hematological abnormalities among their patients with pulmonary TB included normochromic normocytic anemia, thrombocytosis (23). In our study, the total white blood cells (WBC) count was higher $(\mathrm{p}<0.01)$ before treatment, which is in agreement with many previous studies. Neutrophil composition was higher when compared after treatment, and it could be concluded that leukocytosis due to neutrophils is associated with TB (24). No significant increase was in the lymphocyte count in TB patients before treatment $(\mathrm{p}<0.0001)$. However, there were some reports of lymphocytosis and lymphopenia. Lymphocytic reaction reported in pulmonary tissue decays nonspecific inflammation. Many authors reported lymphocytopenia, leucopenia, neutropenia in tuberculosis (21). Data resulting from this study showed that the mean platelet count did not show a significant increase, which is in contrast with other studies by Kartaloglu et al., Venom, AL-Omar et al., and Awodu et al., who found that there was a significantly lower platelet count in African pulmonary tuberculosis patients $(4,19)$. Erythrocyte sedimentation rate at 30 and 60 minutes of pretreatment patients' mean value showed a highly significant increase with normal values $(p<0.01)$. In relation to ESR, some studies have shown that values were higher at pretreatment; as the beginning of the tuberculosis process, there is strong pro-inflammatory cytokine activity (IFN- $\gamma$ and TNF- $\alpha$ ), which stimulates the expression of acute-phase proteins and thrombocytosis. With disease evolution, which promotes cooling down of inflammatory activity $(4,25)$. Also Ukpe et al. found that active TB is associated mostly with very high ESR values $(\geqslant 100 \mathrm{~mm} / \mathrm{h}$ ) regardless of HIV condition, and that concomitant HIV infection tends to increase the proportion of TB cases with these very high values (26). In this study, after infection with M. tuberculosis, the aminotransferases (ALT, AST) levels significantly increased. The increment of liver enzyme levels did not reflect the bacterial load present. Drug treatment lead to a significant decrease of bacterial numbers, especially in combined drug treatment groups $(9,27)$. This finding was similar to that of Preeti Dharmik et al., who reported that, during anti-tuberculosis therapy, the level of the liver is elevated from its normal range, hence causing toxicity or malfunction of the human liver (28). Ashish Anantrao et al. showed that the ALP was used for distinguishing between tuberculosis and other types of pleural effusion (29). Hematological and biochemical indices in pulmonary tuberculosis are common and may be valuables to detect disease. Changes in some hematological parameters may reflect response to treatments (23). There are useful indices of severity in tuberculosis, and the return of these indicators to a normal level is a good symptom of disease control in that they are correlated with sputum exchange to negative acid-fast bacilli (19). There are some parts that may be improved in our retrospective study. The results obtained are clearly not representative of all tuberculosis patients, and the outcomes of all patients are not included because of deficient patient records. Another limitation is interactions between anti-TB drugs and other drugs in patients with comorbidity.

\section{Conclusions}

In summary, this study shows that hematologic parameters and liver enzyme changes in pulmonary TB patients, which is associated with anti-TB drugs. After using drug treatments, hematological parameters (RBC, Hb, HCT, $\mathrm{MCV}, \mathrm{MCH}$, and $\mathrm{MCHC}$ ), except platelet count were changed significantly. Liver enzyme activities (ALT, AST, and ALP) were decreased significantly $(\mathrm{p} \leq 0.001)$ after treatment. The anti-tuberculosis drugs impose a hazardous effect on the patient's liver, leading to hepatotoxicity, and are a major problem in tuberculosis patients. This can be useful in determining response to treatment progress. Prospective studies are necessary to confirm this relationship.

\section{Acknowledgments:}

This study was supported by the Student Research Committee, Ahvaz Jundishapur University of Medical Sciences (NO: 94S13). We also would like to thank the patients for their participation in our study and the hospital staff who assisted in the recruitment and follow-up of patients during the entire study duration.

\section{Conflict of Interest:}

There is no conflict of interest to be declared.

Authors' contributions:

All authors contributed to this project and article equally. All authors read and approved the final manuscript.

\section{References:}

1) Baddeley A, Dean A, Monica-Dias H, Falzon D, Floyd K, Garcia I. Global Tuberculosis Report 2013. World Health Organization. 2013; 306. 
2) Shafee M, Abbas F, Ashraf M, Mengal MA, Kakar N, Ahmad Z, et al. Hematological profile and risk factors associated with pulmonary tuberculosis patients in Quetta, Pakistan. Pak J Med Sci. 2014; 30(1): 36-40. doi: 10.12669/pjms.301.4129. PMID: 24639827, PMCID: PMC3955538.

3) Shih TY, Pai CY, Yang P, Chang WL, Wang NC, Hu OY. A novel mechanism underlies the hepatotoxicity of pyrazinamide. Antimicrob Agents Chemother. 2013; 57(4): 1685-90. doi: 10.1128/AAC.01866-12. PMID: 23357778, PMCID: PMC3623344.

4) Al-muhammadi MO, Hayder A-S. Studying Some Hematological Changes in Patients with Pulmonary Tuberculosis in Babylon Governorate. Medical Journal of Babylon. 2011; 8(4).

5) Sonika U, Kar P. Tuberculosis and liver disease: management issues. Trop Gastroenterol. 2012; 33(2): 102 6. doi: 10.7869/tg.2012.25. PMID: 23025055.

6) Rattan A, Kalia A, Ahmad N. Multidrug-resistant Mycobacterium tuberculosis: Molecular perspectives. Emerg Infect Dis. 1998; 4(2): 195-209. doi: 10.3201/eid0402.980207. PMID: 9621190, PMCID: PMC2640153.

7) Chen Y, Tang Y, Guo C, Wang J, Boral D, Nie D. Nuclear receptors in the multidrug resistance through the regulation of drug-metabolizing enzymes and drug transporters. Biochem Pharmacol. 2012; 83(8): 111226. doi: 10.1016/j.bcp.2012.01.030. PMID: 22326308, PMCID: PMC3339266.

8) Merza MA, Farnia P, Tabarsi P, Khazampour M, Masjedi MR, Velayati AA. Anti-tuberculosis drug resistance and associated risk factors in a tertiary level TB center in Iran: a retrospective analysis. J Infect Dev Ctries. 2011; 5(7): 511-9. doi: 10.3855/jidc.1259. PMID: 21795819.

9) Lenaerts AJ, Johnson CM, Marrieta KS, Gruppo V, Orme IM. Significant increases in the levels of liver enzymes in mice treated with anti-tuberculosis drugs. Int J Antimicrob Agents. 2005; 26(2): 152-8. doi: 10.1016/j.ijantimicag.2005.04.011. PMID: 15953708.

10) Oliva VM, Cezario GAG, Cocato RA, Marcondes-Machado J. Pulmonary Tuberculosis: Hematology, Serum Biochemistry and the Relation with the Disease Duration. Biotechnology. 2008; 14(1): 71-8. doi: 10.1590/S1678-91992008000100006.

11) Sharma SK. Antituberculosis drugs and hepatotoxicity. Infect Genet Evol. 2004; 4(2): 167-70. doi: 10.1016/j.meegid.2003.01.001. PMID: 15157635 .

12) Shirali S, Zahra Bathaie S, Nakhjavani M. Effect of Crocin on the Insulin Resistance and Lipid Profile of Streptozotocin - Induced Diabetic Rats. Phytother Res. 2013; 27(7): 1042-7. doi: 10.1002/ptr.4836. PMID: 22948795.

13) Ebrahimi E, Shirali S, Talaei R. The Protective Effect of Marigold Hydroalcoholic Extract in STZ-Induced Diabetic Rats: Evaluation of Cardiac and Pancreatic Biomarkers in the Serum. Journal of Botany. 2016; 6. doi: $10.1155 / 2016 / 9803928$.

14) Hosseini A, Gorjian M, Rasouli L, Shirali S. A Comparison between the Effect of Green Tea and Kombucha Prepared from Green Tea on the Weight of Diabetic Rats. Biosceces Biotechnology Research Asia. 2015; 12: 141-6. doi: 10.13005/bbra/1616.

15) Barari AR, Alavi H, Shirali S, Ghanaian F. Effect of short-term endurance training and silymarin consumption on some of preinflammatory cytokines, growth mediators and immune system performance. Annals of Biological Research. 2012; 3(6): 2933-7.

16) Akpan Patience A, Akpotuzor Josephine O, Akwiwu Ephoria C. Some haematological parameters of tuberculosis infected Nigerians: A study in Calabar-Nigeria. Global Journal of Pure and Applied Sciences. Bachudo Science Company Limited. 2013; 19(1): 95-100.

17) Taramian S, Joukar F, Asgharnezhad M, Biabani A, Mansour Ghanaei F. Side Effects of First-line Anti Tuberculosis Drugs. Journal of Guilan University of Medical Sciences. 2012; 85(2): 42-7.

18) Khalili H, Dashti-Khavidaki S, Rasoolinejad M, Rezaie L, Etminani M. Anti-tuberculosis drugs related hepatotoxicity; incidence, risk factors, pattern of changes in liver enzymes and outcome. DARU- Journal of Faculty of Pharmacy, Tehran University of Medical Sciences. 2009; 17(3): 163-7..

19) Shareef HA. Abnormalities of hematological parameters in newly diagnosed Pulmonary tuberculosis patients in Kirkuk city. Pakistan journal of medical sciences. 2012; 20(5): 1486-92.

20) Kumar S, Saxena K, Pharm IJ, Bio S, Singh UN, Saxena R. Comparative study of acute proteins in case of Anemia of Chronic Disease (ACD) and Iron Deficiency Anemia (IDA) and its relationship whit erythropoietin. International Journal of Pharmacy and Biological Sciences. 2013; 3(3): 323-8.

21) Thatoi PK, Khadanga S. Pulmonary Tuberculosis and its hematological correlates. Transworld Medical Journal environment. 2013; 7(1): 8.

22) Yaranal PJ, Umashankar T, Govindareddy S. Hematological Profile in Pulmonary Tuberculosis. Int J Health Rehabil Sci. 2013; 2(1): 50-5. 
23) Muzaffar TM, Shaifuzain AR, Imran Y, Haslina MN. Hematological changes in tuberculous spondylitis patients at the Hospital Universiti Sains Malaysia. Southeast Asian J Trop Med Public Health. 2008; 39(4): 686-9. PMID: 19058606.

24) Al-Omar I, Al-Ashban R, Shah A. Hematological abnormalities in Saudis suffering from pulmonary Tuberculosis and their response to the treatment. Research journal of pharmacology. 2009; 3(4): 78-85.

25) Kannan S, Kumar S. Hematological Observation in Tuberculosis and their Response to the Treatment. Journal of Academic and Applied Studiesournal of Hospital and Clinical Pharmacy. 2011; 1(4): 19-24.

26) Ukpe IS, Southern L. Erythrocyte sedimentation rate values in active tuberculosis with and without HIV co-infection. S Afr Med J. 2006; 96(5): 427-8. PMID: 16751918.

27) Mugusi S, Ngaimisi E, Janabi M, Minzi O, Bakari M, Riedel KD, et al. Liver enzyme abnormalities and associated risk factors in HIV patients on efavirenz-based HAART with or without tuberculosis coinfection in Tanzania. PLoS ONE. 2012; 7(7): 1-9. doi: 10.1371/journal.pone.0040180. PMID: 22808112, PMCID: PMC3394799.

28) Dharmik P, Gomashe A, Dolas R, Dhargave T. Effect of antituberculosis treatment on human liver. International journal of Pharma Sciences and Research (IJPSR). 2013; 4(2): 5-9.

29) Jadhav AA, Bardapurkar JS, Jain A. Alkaline phosphatase: Distinguishing between tuberculous and nontuberculous pleural effusion. Lung India. 2009; 26(3): 77-80. doi: 10.4103/0970-2113.53230. PMID: 20442841, PMCID: PMC2862511. 